Pacific Journal of Mathematics

BOUNDEDNESS PRINCIPLES AND FOURIER THEORY 


\title{
BOUNDEDNESS PRINCIPLES AND FOURIER THEORY
}

\author{
R. E. EDWARDS
}

A systematic application of simple functional analytic techniques (boundedness principles and the Hahn-Banach theorem) is made to establish a few results in harmonic analysis.

For the circle group, results are obtained about the possible misbehaviour of conjugate functions and related multiplier transforms.

For infinite compact Abelian groups, results are obtained about the possible misbehaviour of functions or pseudomeasures $f$ for which the Fourier transform $\hat{f}$ is $o(\rho)$, where $\rho$ is a preassigned nonnegative function on the character group.

1. Concerning conjugate functions. Except in Remark (e) at the end of this section, all functions and distributions appearing this section are assumed to have period $2 \pi$, and may thus be regarded as functions and distributions on the circle group. The $n$-th Fourier coefficient of any such (integrable) function or distribution $f$ will be denoted by $\hat{f}(n)$, $n$ here ranging over the set $Z$ of integers.

It is known from the work of Lusin and Tolstov (see [1], Vol. 2, pp. 95-98) that

(i) There exists an absolutely continuous function $f$ whose conjugate function $\tilde{f}$ is essentially unbounded on every nondegenerate interval;

(ii) The function $f$ referred to in (i) may be so chosen that the Fourier series of $f$ and of $\tilde{f}$ are each pointwise convergent a.e..

The Lusin-Tolstov approach is constructive and we have nothing to add to it. Instead, we present an existential proof of (i). Although the proof is nonexplicit, it sheds some light on the underlying reasons for the occurrence of the phenomena concerned.

In discussing (i) we use the fact that, at least for (measurable) functions $f$ such that $f \cdot \log ^{+}|f| \in L^{1}$, the traditionally-defined conjugate function $\widetilde{f}$ may be identified distributionally with $H * f$, where $H$ is the distribution

$$
\sum_{n \in Z}(-i \cdot \operatorname{sgn} n) e^{i n x},
$$

the series being distributionally convergent. It will be convenient to refer to $H$ as the (periodic) Hilbert distribution.

In view of the following theorem, the crucial property of $H$ leading to the phenomenon (i) is seen to be expressed by the formula 


$$
H=2 D\left(\log \left|\sin \frac{1}{2} x\right|\right)=D \phi_{0},
$$

where the derivation symbol $D$ is understood in its distributional sense.

THEOREM 1. Let $K$ be any distribution which is not of the form

$$
K=c+D_{\phi}
$$

$c$ denoting a constant and $\phi \in L^{\infty}$. Then there exist absolutely continuous functions $f$ such that $K * f$, if a function at all in the distributional sense, is essentially unbounded on every nondegenerate interval. (The set of $f$ with the stated property will indeed form a comeagre subset of the space of absolutely continuous functions, the latter being regarded as a Banach space in the fashion described in the proof to follow.)

Proof. Denote by $E$ the space of absolutely continuous functions $f$, and introduce into $E$ the norm

$$
\|f\|_{E}=|f(0)|+\|D f\|_{3} .
$$

It is a simple task to verify that $E$ is complete for this norm and is therefore a Banach space.

Enumerate as $\left(I_{r}\right)_{r=1}^{\infty}$ all nonvoid open subintervals of $[0,2 \pi]$ with rational endpoints, and observe that any nondegenerate subinterval of $[0,2 \pi]$ contains $I_{r}$ for some $r$. For each $r$ define the function $N_{r}$ from $E$ into $[0, \infty]$ by

$$
N_{r}(f)=\operatorname{Sup}|\langle\theta, K * f\rangle|,
$$

the supremum being taken with respect to all periodic $C^{\infty}$ functions $\theta$ whose support is contained in $I_{r}$ and which satisfy $\|\theta\|_{1} \leqq 1$.

Adopting the usual conventions:

$$
\begin{aligned}
\alpha+\infty & =\infty+\infty=\infty & & \text { if } 0 \leqq \alpha \leqq \infty, \\
\alpha \cdot \infty & =\infty & & \text { if } 0<\alpha \leqq \infty, 0 \cdot \infty=0,
\end{aligned}
$$

it is very simple to check that

$$
\left\{\begin{array}{l}
N_{r}(f+g) \leqq N_{r}(f)+N_{r}(g), \\
N_{r}(\lambda f)=|\lambda| \cdot N_{r}(f),
\end{array}\right\}
$$

for $f, g \in E$ and $\lambda$ a scalar.

To this must be added the observation that if $f \in E$ is such that $N_{r}(f)<\infty$, then the distribution $K * f$ coincides on $I_{r}$ with a function 
in $L^{\infty}$ whose $L^{\infty}$-norm does not exceed $N_{r}(f)$. This follows from the definition of $N_{r}(f)$, the Hahn-Banach theorem, and the known form of the dual of any $L^{1}$-space (see, for example, [2], Theorem 4.16.1).

As a final preliminary step, we show that each $N_{r}$ is lower semicontinuous on $E$, i.e., that the set

$$
S=\left\{f \in E: N_{r}(f) \leqq 1\right\}
$$

is closed in $E$. In fact, if the functions $f_{k}(k=1,2, \cdots)$ belong to $S$ and converge in $E$ to the limit $f$, then (1.3) shows that $f_{k} \rightarrow f$ uniformly. Therefore $K * f_{k} \rightarrow K * f$ distributionally. Using the substance of the preceding paragraph, it follows that $K * f$ coincides on $I_{r}$ with some $L^{\infty}$-function whose $L^{\infty}$-norm does not exceed 1 ; in other words, $f \in S$.

Suppose now that Theorem 1 were false. In view of what we know about the $N_{r}$, a known boundedness principle ([2], Theorem 7.5.1) would then entail that an integer $r$ exists such that $N_{r}$ is finite-valued and continuous on $E$, i.e., that there exists an $f$-independent number $k$ such that

$$
N_{r}(f) \leqq k \cdot\|f\|_{E}
$$

for all $f \in E$. Choosing $a \in I_{r}$ and translating $f$ by amount $a$, it would appear that, for all trigonometric polynomials $f$,

$$
|\langle f, K\rangle| \leqq k \cdot\left\{|f(a)|+\|D f\|_{1}\right\} .
$$

Let $T$ denote the linear mapping of the space $P$ of trigonometric polynomials into the product space $\Phi \times L^{1}$ ( $\Phi$ the scalar field) defined by $T f=(f(a), D f)$ and define the linear functional $\Gamma$ on $T(P)$ by the formula $\Gamma(T f)=\langle f, K\rangle$. Then (1.5) would imply that $\Gamma$ is continuous on the subspace $T(P)$ of $\Phi \times L^{1}$ and would therefore, by the Hahn-Banach theorem, admit a continuous extension to this product space. As a consequence one would have a representation formula

$$
\langle f, K\rangle=\Gamma(T f)=c \cdot f(a)-(1 / 2 \pi) \int_{0}^{2 \pi} D f \cdot \dot{\phi}_{1} \cdot d x,
$$

wherein $c$ is a scalar and $\dot{\phi}_{1} \in L^{\infty}$.

In distributional notation, this would signify that

$$
\langle f, K\rangle=c \cdot\left\langle f, \varepsilon_{a}\right\rangle+\left\langle f, D \dot{\phi}_{1}\right\rangle
$$

where $\varepsilon_{a}$ denotes the Dirac measure at $a$, so that

$$
K=c \cdot \varepsilon_{a}+D \phi_{1} .
$$

Adding to $\phi_{1}$ a function of bounded variation whose distributional derivative is equal to $c\left(\varepsilon_{a}-1\right)$, one would obtain a function $\phi \in L^{\infty}$ 
for which the forbidden formula (1.2) would hold. This contradiction establishes Theorem 1.

REMARKs. (a) The preceding proof shows indeed that there exist absolutely continuous functions $f$ with the following property: for each nondegenerate subinterval $I$ of $[0,2 \pi]$, either $K * f$ agrees distributionally on $I$ with no (integrable) function, or $K * f$ does so agree on $I$ with an (integrable) function which is essentially unbounded on $I$.

(b) In applying Theorem 1 to $K=H$ in order to derive (i), observe that $H * f$ is obviously a function in $L^{2}$ whenever $f \in L^{2}$.

It may also be remarked that the fact that $H$ is not a measure is, in a similar way, the root reason why there exist integrable functions $f$ such that $H * f$ is not integrable, and continuous functions $f$ such that $H * f$ is not continuous.

(c) Since $f$ is absolutely continuous if and only if $f$ is continuous and $D f \in L^{1}$, it is worth noting that there is an analogue of Theorem 1 applying to functions $f$ such that $f$ is continuous and $D f \in L^{p}$ for some $p>1$. This analogue asserts the existence of such functions $f$ for which

$$
\limsup _{a \rightarrow 0} \omega_{\infty} \widetilde{f}(a) / \omega(\alpha)=\infty \quad \text { as } a \downarrow 0
$$

whenever $\omega(\alpha)$ is a preassigned function, bounded away from zero with $a$, and such that

$$
\omega(a)=o\left(a^{1-1 / p}\right)
$$

(Here $\omega_{\infty} \tilde{f}(a)=\operatorname{Sup}_{x}|\widetilde{f}(x-a)-\widetilde{f}(x)|$.) A possible proof proceeds along the lines of that of Theorem 1. Alternatively, as was pointed out by a referee, one can proceed on the basis of the fact that, for $1<p<\infty$, the Hilbert transform is an automorphism of the subspace

$$
L_{0}^{p}=\left\{f \in L^{p}: \hat{f}(0)=0\right\}
$$

of $L^{p}$, while for a given $a$ the functional

$$
f \rightarrow \int_{0}^{a} f(x) d x
$$

has a norm equal to $a^{1-1 / p}$ when acting on $L_{0}^{p}$.

(d) Theorem 1 shows that the multiplier operators from absolutely continuous functions to $L^{\infty}$ (or to the continuous functions) are precisely those obtained by convolution with a distribution of the form (1.2).

(e) It is perhaps worth noting that Theorem 1 has an analogue for the case in which the circle group is replaced by the additive group $R$ of real numbers and restrictions of periodicity are dropped, 
namely: Suppose that $K$ is a distribution on $R$ which is not of the form

$$
K=\psi+D \dot{\phi},
$$

where $\phi$ and $\psi$ belong to $L^{\infty}$. Then there exists an absolutely continuous function $f$ which tends to zero at infinity, for which $f$ and $D f$ belong to $L^{1}$, and for which $K * f$, if a function at all, is essentially unbounded on every nondegenerate subinterval of $R$. (The proof follows the same lines as does that of Theorem 1.)

This result applies in particular when

$$
K=D(\log |x|)=F \cdot P \cdot|x|^{-1},
$$

in which case $K * f=\tilde{f}$ is the one-dimensional Hilbert transform of $f$.

2. Results for compact Abelian groups. From statement (i) of $\S 1$ follows the existence of functions $f$ (necessarily in $L^{2}$ ) on the circle group such that

$$
\widehat{f}(n)=o(1 / n) \quad \text { as } n \rightarrow \infty,
$$

and yet $f$ is essentially unbounded on every nondegenerate interval. In this section we shall obtain a result which is at once more precise and more general.

It is assumed throughout this section that $G$ denotes an infinite, compact (Hausdorff), Abelian group, while $\left(\Omega_{r}\right)_{r=1}^{\infty}$ denotes a sequence of nonvoid open subsets of $G$. (The term "measurable" refers to normalised Haar measure on $G$, and $L^{p}$ will denote the associated Lebesgue space.) One may, if $G$ is first-countable, choose the $\Omega_{r}$ to form a countable open base for $G$. Furthermore, $\rho$ will denote a nonnegative valued function on the (infinite discrete) character group $X$ of $G$. Summation signs which are otherwise unspecified will refer to summation over $X$.

THEOREM 2. To each $\rho$ satisfying

$$
\Sigma \rho=\infty
$$

corresponds at least one function $f$ on $G$ such that

(i) $f \in L^{p}$ for every $p<\infty$;

(ii) $f$ is essentially unbounded on $\Omega_{r}$ for every $r$;

$$
\text { (iii) } \hat{f}(\chi) \equiv \int_{G} f(x) \overline{\chi(x)} d x=o(\rho(\chi)) \text { as } \chi \rightarrow \infty \text {. }
$$

Proof. A preliminary argument, the details of which are omitted, shows that any $\rho$ satisfying (2.1) can be replaced by a nonnegative 
function $\rho^{\prime}$ which is $O(\rho)$, which satisfies (2.1), and for which

$$
\sum \rho^{\prime+\varepsilon}<\infty \quad \text { for every } \varepsilon>0 \text {. }
$$

We may as well, therefore, assume that

$$
\sum \rho^{1+\varepsilon}<\infty \quad \text { for every } \varepsilon>0 .
$$

Let $X_{0}=\{\chi \in X: \rho(\chi) \neq 0\}$.

Denote by $E$ the linear space of functions $f$ which belong to $L^{p}$ for every finite $p$, which satisfy $\hat{f}\left(X \backslash X_{0}\right) \subset\{0\}$, and which satisfy (2.2). Define on $E$ the norms

$$
N_{p}(f)=\|f\|_{p}+\|f\|^{*} \quad(p=1,2, \cdots)
$$

where

$$
\|f\|^{*}=\operatorname{Sup}_{\chi \in x_{0}}|\hat{f}(\chi)| / \rho(\chi)
$$

Endowed with the topology having as base at 0 the sets

$$
U_{p, \varepsilon}=\left\{f \in E: N_{p}(f)<\varepsilon\right\},
$$

where $p$ ranges over the positive integers and $\varepsilon$ over the positive numbers, $E$ is easily seen to be a Fréchet space.

Define further the functions

$$
M_{r}(f)=\underset{x \in \Omega_{r}}{\operatorname{ess} \sup _{r}}|f(x)|,
$$

where $r=1,2, \cdots$. Each $M_{r}$ has the properties expressed in (1.4) and is further lower semicontinuous on $E$.

Were Theorem 2 to be false, to each $f \in E$ would correspond an integer $r=r(f)$ such that $M_{r}(f)<\infty$. Then, by Theorem 7.5.1 of [2], there would exist an $f$-independent integer $r$ such that $M_{r}$ is finite-valued and continuous on $E$. In other words, there would exist for this $r$ a number $k>0$ and an exponent $p<\infty$ such that

$$
M_{r}(f) \leqq k\left\{\|f\|_{p}+\|f\|^{*}\right\} \text {. }
$$

Since $E$ is evidently translation-invariant, and since $Q_{r}$ in nonvoid and open, this inequality would combine with compactness of $G$ to entail that

$$
\|f\|_{\infty} \leqq k\left\{\|f\|_{p}+\|f\|^{*}\right\}
$$

for each $f \in E$.

As a consequence of (2.4) it would appear that, for each $g \in L^{1}$, the linear functional 


$$
f \rightarrow \int_{\theta} f \breve{g} d x \quad\left(\breve{g}(x) \equiv g\left(x^{-1}\right)\right)
$$

is defined and continuous on $E$. By an argument similar to that used at the corresponding stage in the proof of Theorem 1, it would follow that there exists $h \in L^{p^{\prime}}$ and $\alpha \in l^{1}\left(X_{0}\right)$ such that

$$
\int_{a} f \breve{g} d x=\int_{a} f \breve{h} d x+\sum_{x_{0}} \alpha(\chi) \hat{f}(\chi) / \rho(\chi)
$$

for each $f \in E$, so that, in particular,

$$
\rho \hat{g}=\rho \hat{h}+\alpha,
$$

provided we define $\alpha(\chi)=0$ for $\chi \in X \backslash X_{0}$.

Now $p^{\prime}>1$ and, by considering separately the cases in which $1<p^{\prime}<2$ and $p^{\prime} \geqq 2$, the Hausdorff-Young and Parseval inequalities would lead from (2.3) and (2.5) to the conclusion that

$$
\sum \rho|\hat{g}|<\infty
$$

for each $g \in L^{1}$. An application of Corollary 7.1.2 of [2] would then yield the result

$$
\sum \rho|\hat{g}| \leq \text { const. }\|g\|_{1} \text {. }
$$

Finally, on letting $g$ range over an approximate identity in $L^{1}$, (2.6) would lead to a contradiction of (2.1), and thus to an indirect proof of Theorem 2.

Remarks. (a) If $G$ is the circle group (so that $X=Z$ ), and if it be assumed that

$$
\limsup _{|n| \rightarrow \infty}|n| \rho(n)<\infty,
$$

then (2.2) ensures that the Fourier series of $f$ is a.e. pointwise convergent (as follows from Fatou's theorem; see [1], Vol. 1, p. 178).

(b) Things can be made more explicit, if it is given that

$$
\sum_{S} \rho=\infty
$$

for some Sidon subset $S$ of $X$ (see [3], 5.7); here $\sum_{S}$ denotes summation extended over $S$. In this case one may assume without loss of generality that in addition

$$
\sum_{S} \rho^{2}<\infty,
$$

and it then follows from 5.7.3 and 5.7.7 of [3] that, on choosing any function $\theta \in c_{0}(X)$, the space of complex-valued functions on $X$ which 
tend to zero at infinity, for which

$$
\sum_{S} \rho \cdot \theta=\infty,
$$

the function

$$
f_{\theta}(x)=\sum_{\vec{S}} \rho(\chi) \theta(\chi) \chi(x)
$$

satisfies (i) of Theorem 2 and does not belong to $L^{\infty}$; and it is evident that $f_{\theta}$ satisfies (iii) of Theorem 2 .

An application of the boundedness principle to the space of functions $f_{\theta}$, obtained when $\theta$ ranges over $c_{0}(X)$, shows that $\theta$ may be chosen so that $f_{\theta}$ satisfies condition (ii) of Theorem 2 .

A referee has kindly pointed out that the known properties of random Fourier series may also be used to much the same end.

(c) It is known that if

$$
\sum \rho^{2}=\infty,
$$

then there exist functions $\omega$ on $X$ which are $o(1)$ and such that $\sum \omega(\chi) \rho(\chi) \chi(x)$ is not the Fourier-Stieltjes series of any measure on G.

Supposing that $G$ is first countable, the proof of Theorem 2 can be modified so as to prove more, namely: Suppose that $\rho$ satisfies (2.7), that $1 \leqq p<\infty$, and that $\alpha$ is a nonnegative function on $X$ such that

$$
\begin{cases}\alpha \in l^{q}(X) \text { for some } q<2 /(2-p) & \text { if } 1 \leqq p<2 \\ \alpha \in l^{\infty}(X) & \text { if } 2 \leqq p<\infty\end{cases}
$$

then there exists a pseudomeasure $\sigma$ on $G$ (see [4], [5], [6]) such that

$$
\left\{\begin{array}{l}
\operatorname{sing} \operatorname{supp} \sigma=G, \hat{\sigma}=o(\rho) \\
\alpha^{1 / p} \hat{\sigma} \in l^{p}(X)
\end{array}\right.
$$

When $p=2$, the final clause of (2.9) means that $\sigma$ has "finite energy" in a sense which depends upon the choice of $\alpha$.

\section{REFERENCES}

1. N. Bary, A Treatise on Trigonometric Series, Vols. $1 \& 2$, Pergamon Press, 1964.

2. R. E. Edwards, Functional Analysis: Theory \& Applications, Holt, Rinehart \& Winston, Inc., 1965.

3. W. Rudin, Fourier Analysis on Groups, Interscience Publishers, New York, 1962.

4. J.-P. Kahane, and R. Salem, Ensembles parfaits et séries trigonométriques, Hermann et Cie., Paris, 1963. 
5. J.-P. Kahane, Transformées de Fourier des fonctions sommables, Proc. Int. Congress Math. Stockholm (1962), 114-131.

6. R. E. Edwards, Supports and singular supports of pseudomeasures, J. Austral. Math. Soc. VI (1966), 65-75.

Received August 20, 1965.

Institute of AdVanced Studies

AUSTRALIAN NATIONAL UNIVERSITY 



\section{PACIFIC JOURNAL OF MATHEMATICS}

\section{EDITORS}

H. SAMELSON

Stanford University

Stanford, California

J. P. JANS

University of Washington

Seattle, Washington 98105

\section{J. DugunduI}

University of Southern California Los Angeles, California 90007

RICHARD ARENS

University of California

Los Angeles, California 90024

\section{ASSOCIATE EDITORS}
E. F. BECKENBACH
B. H. NEUMANN
F. WOLF
K. YoSIDA

\section{SUPPORTING INSTITUTIONS}

\author{
UNIVERSITY OF BRITISH COLUMRIA \\ CALIFORNIA INSTITUTE OF TECHNOLOGY \\ UNIVERSITY OF CALIFORNIA \\ MONTANA STATE UNIVERSITY \\ UNIVERSITY OF NEVADA \\ NEW MEXICO STATE UNIVERSITY \\ OREGON STATE UNIVERSITY \\ UNIVERSITY OF OREGON \\ OSAKA UNIVERSITY \\ UNIVERSITY OF SOUTHERN CALIFORNIA
}

\author{
STANFORD UNIVERSITY \\ UNIVERSITY OF TOKYO \\ UNIVERSITY OF UTAH \\ WASHINGTON STATE UNIVERSITY \\ UNIVERSITY OF WASHINGTON \\ AMERICAN MATHEMATICAL SOCIETY \\ CHEVRON RESEARCH CORPORATION \\ TRW SYSTEMS \\ NAVAL ORDNANCE TEST STATION
}

Mathematical papers intended for publication in the Pacific Journal of Mathematics should be typewritten (double spaced). The first paragraph or two must be capable of being used separately as a synopsis of the entire paper. It should not contain references to the bibliography. Manuscripts may be sent to any one of the four editors. All other communications to the editors should be addressed to the managing editor, Richard Arens at the University of California, Los Angeles, California 90024 .

50 reprints per author of each article are furnished free of charge; additional copies may be obtained at cost in multiples of 50 .

The Pacific Journal of Mathematics is published monthly. Effective with Volume 16 the price per volume (3 numbers) is $\$ 8.00$; single issues, $\$ 3.00$. Special price for current issues to individual faculty members of supporting institutions and to individual members of the American Mathematical Society: $\$ 4.00$ per volume; single issues $\$ 1.50$. Back numbers are available.

Subscriptions, orders for back numbers, and changes of address should be sent to Pacific Journal of Mathematics, 103 Highland Boulevard, Berkeley 8, California.

Printed at Kokusai Bunken Insatsusha (International Academic Printing Co., Ltd.), No. 6, 2-chome, Fujimi-cho, Chiyoda-ku, Tokyo, Japan.

\section{PUBLISHED BY PACIFIC JOURNAL OF MATHEMATICS, A NON-PROFIT CORPORATION}

The Supporting Institutions listed above contribute to the cost of publication of this Journal, but they are not owners or publishers and have no responsibility for its content or policies. 


\section{Pacific Journal of Mathematics}

\section{Vol. 21, No. 2 December, 1967}

Arne P. Baartz, The measure algebra of a locally compact semigroup ..... 199

Robert F. Brown, On maps with identical fixed point sets............. 215

C. Buttin, Existence of a homotopy operator for Spencer's sequence in the analytic case ..................................... 219

Henry Werner Davis, An elementary proof that Haar measurable almost periodic functions are continuous ........................ 241

Zeev Ditzian, On asymptotic estimates for kernels of convolution transforms ...................................... 249

Robert E. Edwards, Boundedness principles and Fourier theory ......... 255

John A. Hildebrant, On compact unithetic semigroups ............... 265

Marinus A. Kaashoek and David Clark Lay, On operators whose Fredholm set is the complex plane ............................ 275

Sadao Kató, Canonical domains in several complex variables ........... 279

David Clifford Kay, The ptolemaic inequality in Hilbert geometries.... . . . 293

Joseph D. E. Konhauser, Biorthogonal polynomials suggested by the Laguerre polynomials ............................. 303

Kevin Mor McCrimmon, Macdonald's theorem with inverses .......... 315

Harry Eldon Pickett, Homomorphisms and subalgebras of multialgebras .................................... 327

Richard Dennis Sinkhorn and Paul Joseph Knopp, Concerning nonnegative matrices and doubly stochastic matrices ..............

Erling Stormer, On anti-automorphisms of von Neumann algebras ...

Miyuki Yamada, Regular semi-groups whose idempotents satisfy permutation identities .......................... 